\title{
SULLA DIFFUSIONE DEGLI ELETTRONI NELLA IONOSFERA
}

\author{
F. Mariani
}

Il problema della diffusione è stato a varie riprese affrontato da varii autori $\left({ }^{1}\right)\left({ }^{2}\right)\left({ }^{3}\right)\left({ }^{4}\right)$; in particolare Ferraro ha mostrato cone, supponendo costante con la quota la temperatura e considerando quale unico processo di scomparsa degli elettroni la ricombinazione con gli ioni positivi, la diffusione risulta del tutto trascurabile negli strati $E$ e $F_{1}$ mentre, sebbene non del tutto trascurabile nello strato $F_{2}$, essa influisce solo moderatamente sulla sua frequenza critica; Yonezawa invece ha studiato, nelle stesse ipotesi, il caso della scomparsa per attaccamento e ha indicato in $5 \cdot 10^{9} \cdot \mathrm{cm}^{-3}$ la densità minima di particelle nello strato $F_{2}$.

Ora in realtà si ammette generalmente che nello strato $F_{\text {o }}$ in particolare la temperatura vada crescendo con la quota, cosicché occorre prendere in considerazione anche l'effetto della diffusione termica. Nella presente nota, che si riconnette strettamente ad altre note nelle quali l'autore ha studiato sotto varî aspetti l'andamento della intensità di ionizzazione e della densità elettronica in atmosfera non isoterma, esaminiamo appunto il problema della diffusione in una atmosfera non isoterma nell'ipotesi che ciascuno strato ionizzato sia elettricamente neutro e quindi che le densità ionica ed elettronica siano ad ogni quota tra loro uguali e che la temperatura, e quindi la densità di materia, siano ad ogni quota costanti nel tempo, ipotesi questa che, oltre che suggerita da ragioni di semplicità, abbiamo mostrato $\left({ }^{5}\right)$ essere valida almeno nelle ore diurne.

Paragrafo 1. - L'equazione differenziale cui deve ubbidire la densità elettronica $N$ risulta, ovviamente,

$$
\frac{\partial N}{\partial t} \quad I_{0} F-\alpha N^{2}-\operatorname{div}\left(N \overrightarrow{v_{1}}\right)
$$

ove

$I_{0} F=$ intensità di ionizzazione 
$I_{\mathrm{o}}=$ valore massimo $\mathrm{di} I$ per $\underline{\underline{\alpha}}=0^{\circ}$

$\alpha=$ coefficiente $\mathrm{di}$ ricombinazione generalizzato

$\overrightarrow{v_{1}}=$ velocità di diffusione degli elettroni.

Per stabilire la forma del termine $\overrightarrow{N v_{1}}$ supporremo che lo strato ionizzato possa essere considerato come costituito da molecole neutre e da un miscuglio ioni-elettroni che considereremo come un unico gas (in virtù delle forze elettrostatiche attrattive che legano ioni ed elettroni).

Secondo la teoria generale della diffusione $\left({ }^{6}\right)$ per un miscuglio binario (nel nostro caso il gas ioni-elettroni e il gas di molecole neutre) possiamo scrivere, indicando con $\vec{v}_{1}$ e $\overrightarrow{v_{2}}$ le rispettive velocità medie di diffusione,

$$
\begin{gathered}
\vec{v}_{1}-\vec{v}_{2}=-\frac{n^{2}}{n_{1} n_{2}} D_{22}\left[\frac{\partial}{\partial z} \frac{n_{1}}{n}+\frac{n_{1} n_{2}}{n \rho P}\left(m_{2}-m_{1}\right) \frac{\partial P}{\partial z}-k_{\mathrm{T}} \frac{1}{T} \frac{\partial T}{\partial z}-\right. \\
\left.-\frac{\rho_{1} P}{\rho P}\left(\vec{F}_{1}-\vec{F}_{2}\right)\right]
\end{gathered}
$$

Ove

$$
\begin{aligned}
z & =\text { altezza; } \\
n_{1}, n_{2} & =\text { concentrazioni dei due gas; } \\
n & =n_{1}+n_{2} ; \\
m_{1}, m_{2} & =\text { masse molecolari medie dei due gas; } \\
\rho_{1}, \rho_{2} & =\text { densità dei due gas; } \\
\rho & =\rho_{1}+\rho_{2} ; \\
P & =\text { pressione; } \\
T & =\text { temperatura assoluta; } \\
k_{\mathrm{T}} & =\text { coefficiente di diffusione termica; } \\
F_{1}, F_{2} & =\text { forze per unità di massa agenti sulle particelle dei due gas; } \\
D_{12} & =\frac{3}{8 n \sigma_{0}^{2}}\left[\frac{k T\left(m_{1}+m_{2}\right)}{2 \pi m_{1} m_{2}}\right]^{1 / 2}=\text { coefficiente di diffusione; } \\
\sigma_{0} & =\text { raggio della sfera cui si assimilano ioni e molecole; } \\
k & =\text { costante di Boltzmann. }
\end{aligned}
$$


Alla [2] aggiungeremo la condizione

$$
n_{1} \vec{v}_{1}+n_{2} \vec{v}_{9}=0
$$

che esprime appunto il fatto che, in media, non si ha passaggio di particelle attraverso un qualunque piano orizzontale.

Se indichiamo con

$$
T(z)=T_{0} f(z) \quad \text { con } T_{0}=T(0)
$$

la legge di dipendenza della temperatura dalla quota, la densità materiale $\rho$ si scrive nella forma

$$
\rho(z)=\rho_{0} \exp _{\|}^{!}\left[-\frac{1}{H_{n}} \int_{0}^{\mathrm{z}}\left(1+H_{0} \frac{d f}{i z}\right) \frac{d z}{f(z)}\right]
$$

ove $\rho_{0}$ e $H_{0}$ sono la densità e la scala delle altezze alla quota convenzionale $z=0$.

Se i due gas sono soggetti alla sola azione della gravità, nella [2] si annulla l'ultimo termine; di conseguenza, dalle precedenti relazioni [2] [3] [4] [5] e identificando $n_{1} \operatorname{con} N$ della [1], si deduce l'espressione

$$
N v_{1}=-D_{12}\left[\frac{\partial N}{\partial z}+\left(n_{T}+\cdots n_{\mathrm{T}}\right) \frac{1}{f(z)} \frac{d f}{d z}+\frac{m_{1} g}{k T_{0}} \frac{N}{f(z)}\right]
$$

Il coefficiente $k_{\mathrm{T}}$ può essere scritto nella forma

$$
k_{\mathrm{T}}=\alpha_{\mathrm{T}} \frac{N}{n} \frac{n_{2}}{n}
$$

$\operatorname{con} \alpha_{\mathrm{T}}$ sensibilmente indipendente da temperatura e da concentrazione.

Dalle [6] e [7], imponendo la condizione $\frac{n_{2}}{n} \sim 1$ esprimente che la concentrazione di molecole neutre è assai maggiore di quella del gas di ioni-elettroni, si perviene a scrivere il terzo termine che compare nella [1] nella forma

$$
\frac{d\left(N v_{1}\right)}{d z}=-D_{12}\left[\frac{\partial^{2} N}{\partial z^{2}}+Q_{1} \frac{\partial N}{\partial z}+Q_{2} N\right]
$$

con

$$
Q_{1}=\frac{1}{f(z)}\left[\left(\frac{5}{2}+\alpha_{\mathrm{T}}\right) \frac{d f}{d z}+\frac{1}{H_{10}}+\frac{1}{H_{20}}\right]
$$




$$
\begin{aligned}
Q_{2}= & \frac{1}{[f(z)]^{2}}\left[\left(1+\alpha_{\mathrm{T}}\right) f \frac{d^{2} f}{d z^{2}}+\frac{1}{2}\left(1+\alpha_{\mathrm{T}}\right)\left(\frac{d f}{d z}\right)^{2}+\right. \\
& \left.+\left(\frac{1}{2 H_{10}}+\frac{1+\alpha_{\mathrm{T}}}{H_{20}}\right) \frac{d f}{d z}+\frac{1}{H_{10}} \cdot \frac{1}{H_{20}}\right]
\end{aligned}
$$

ove si sono indicate rispettivamente con

$$
H_{10}=\frac{k T_{0}}{m_{1} g} \text { e } H_{20}=\frac{k T_{0}}{m_{2} g}
$$

le scale delle altezze del gas ioni-elettroni e del gas molecolare alla quota $z=0$; ovviamente, nella [5], si identificherà $H_{0}$ con $H_{20}$ in virtù della condizione $\frac{n_{2}}{n} \sim 1$.

In definitiva si arriva a scrivere l'equazione [1] nella forma

$$
\frac{\partial N}{\partial t}=I_{0} F-\alpha N^{2}+D_{12}\left[\frac{\partial^{2} N}{\partial z^{2}}+\tilde{U}_{1} \frac{\partial N}{\partial z}+\hat{Q}_{2} N\right]
$$

Si constata subito che l'equazione [12], nel caso $T=$ cost cioè $f(z)=1$, si riduce a quella considerata da Ferraro con

$$
\left\{\begin{array}{l}
Q_{1}=\frac{1}{H_{11}}+\frac{1}{H_{91}} \\
Q_{z}=\frac{1}{H_{10}} \cdot \frac{1}{H_{20}}
\end{array}\right.
$$

Considerando invece il caso non isotermo e imponendo per semplicità che la temperatura sia funzione lineare della quota, possiamo scrivere

$$
f(z)=1+p z \quad z>0 ；
$$

in corrispondenza la $I(z)$ assume la forma

$I(z)=I_{0} \exp \left\{\mu\left[1-\sec \chi\left(\frac{1+p z}{1+p z^{*}}\right)^{\mathrm{r}}\right]\right\} \cdot\left(\frac{1+p z}{1+p z^{*}}\right)^{\mathrm{r}-1}$

con $r=-\frac{1}{p H_{20}} ; \mu=1+p H_{20} ; z^{*}=$ quota alla quale $I$ è massima per $\chi=0^{\circ}$ e le [9] e [10] si semplificano riducendosi alle espressioni

$$
Q_{1}=\frac{p}{1+p z} G_{1} \quad \text { con } G_{1}=\frac{5}{2}+\alpha_{T}-\frac{3}{2} r
$$




$$
Q_{2}=\frac{p^{2}}{(1+p z)^{2}} G_{2} \quad \text { con } G_{2}=\frac{1}{2}\left(1+\alpha_{\mathrm{T}}\right)-r\left(\frac{5}{4}+\alpha_{\mathrm{T}}\right)+\frac{r^{2}}{2}
$$

$\left[10^{\prime}\right]$

ove, tenuto conto delle [11] e assunto $m_{1}=\frac{m_{2}}{2}$, si è posto senz'altro $r=-\frac{1}{p H_{20}}=-\frac{2}{p H_{10}}$.

In conclusione, tenuto conto delle [12] [9'] [10'] e [15], la equazione di scomparsa, viene a scriversi, nellipotesi che il coefficiente $\alpha$ sia costante e con le usuali notazioni $\vee=\frac{N}{N_{\mathrm{o}}}, \sigma=\frac{1}{1,37 \cdot 10^{4} N_{\mathrm{o}} \alpha} \cdot N_{\mathrm{o}}=$ $=\sqrt{-.}, \Phi=\frac{2 \pi}{86400} t$ $\sigma \frac{\partial v}{\partial \Phi}=F-v^{2}+\tau\left(1+p z^{*}\right)^{-r-1 / 2} v^{-r+3 / 2}\left[\frac{\partial^{2} v}{\partial v^{*}}+\frac{G_{1}}{v} \frac{\partial v}{\partial v}+\frac{G_{0}}{v^{2}} v\right]$

con

$$
\begin{aligned}
& \tau=1,37 \cdot 10^{\circ} \sigma D_{0} p^{2} ; D_{\mathrm{o}}=D_{12}(z=0) ; \\
& v=\frac{1+p z}{1+p z^{*}}
\end{aligned}
$$

Ove si voglia considerare il caso del semplice attaccamento, o più generalmente il caso in cui $\alpha$, anziché essere costante fosse inversamente proporzionale a $N$, allora la equazione [16] va sostituita dalla equazione

$$
\sigma \frac{\partial \nu}{\partial \Phi}=F-v+\tau\left(1+p z^{*}\right)^{-r-1 / 2} v^{-r+3 l^{2}}\left[\frac{\partial^{2} v}{\partial v^{9}}+\frac{G}{v} \frac{\partial v}{\partial v}+\frac{G_{0}}{v^{2}},\right]
$$

$\operatorname{con} \sigma=\frac{1}{1,37 \cdot 10^{4} \beta}, N_{\mathrm{o}}=\frac{I_{\mathrm{o}}}{\beta}, \beta$ coefficiente di attaccamento e $\tau$ ancora dato dalla [17].

Prima di procedere vogliamo valutare l'ordine di grandezza del parametro $\tau$. Come prima cosa osserviamo che usando l'espressione di $D_{12}$ che si è introdotta nella [2] si vengono a trascurare gli effetti dell'attrazione tra le particelle dei due gas: ora, adottando il modello di Sutherland per tale attrazione, si ottiene un fattore correttivo che, 
per temperature dell'ordine dei $1000^{\circ} \mathrm{K}$ è inferiore ma sensibilmente vicino all'unità; poiché d'altra parte ciò che ci interessa è principalmente l'ordine di grandezza dell'effetto della diffusione noi trascureremo senz'altro la correzione indicata.

Per quanto riguarda gli strati $E$ ed $F_{1}$, se, come spesso si assume, si considerano isotermi, valgono senz'altro i risultati di Ferraro; nel caso non isotermo da noi considerato e ponendo nella espressione del coefficiente di diffusione $i$ valori

$\sigma_{\mathrm{o}}=3,5 \cdot 10^{-8} \mathrm{~cm} ; m_{1}=2,33 \cdot 10^{-23} \mathrm{gr} ; m_{2}=4,66 \cdot 10^{-23} \mathrm{gr}$;

$T=T_{\mathrm{o}} ; n=n(z=o)=n_{\mathrm{o}}$;

si ottiene $D_{0}-3,65 \cdot 10^{17} \frac{T_{0}^{1 / 2}}{n_{0}}$ cosicché, con le ulteriori specificazioni indicate nella tabella che segue, si ottengono, nel caso della ri-

\begin{tabular}{|c|c|c|}
\hline Strato $E$ & Strato $F_{1}$ & Strato $F_{2}$ \\
\hline$n_{0}=5 \cdot 10^{12} \mathrm{~cm}^{-3}$ & $n_{0}=5 \cdot 10^{\mathrm{11}} \mathrm{cm}^{-3}$ & $n_{0}=5 \cdot 10^{10} \mathrm{~cm}^{-3}$ \\
$T_{0}=300{ }^{\circ} \mathrm{K}$ & $T_{0}=500{ }^{\circ} \mathrm{K}$ & $T_{0}=800{ }^{\circ} \mathrm{K}$ \\
$N_{0}=2 \cdot 10^{5} \mathrm{~cm}^{-3}$ & $N_{0}=2 \cdot 10^{5} \mathrm{~cm}^{-3}$ & $N_{0}=10^{8} \mathrm{~cm}^{-3}$ \\
$\operatorname{grad} T=2{ }^{\circ} \mathrm{K} / \mathrm{km}$ & $\operatorname{grad} T=4{ }^{\circ} \mathrm{K} / \mathrm{km}$ & $\operatorname{grad} T=10^{\circ} \mathrm{K} / \mathrm{km}$ \\
$p=\operatorname{grad} \frac{T}{T_{0}}=0,66 \cdot 10^{-7} \mathrm{~cm}^{3}$ & $p=0,8 \cdot 10^{-3} \mathrm{~cm}^{2}$ & $p=1,25 \cdot 10^{-7} \mathrm{~cm}^{-1}$ \\
$\alpha=10^{-8} \mathrm{~cm}^{3} \mathrm{sec}^{-1}$ & $\alpha=10^{-8} \div 10^{-8} \mathrm{~cm}^{3} \mathrm{sec}^{-1}$ & $\alpha=10^{-8} \div 10^{-10} \mathrm{~cm}^{3} \mathrm{sec}^{-1}$ \\
\hline
\end{tabular}

combinazione, per i paramenti $\sigma$ e $\tau$ i seguenti valori

strato $E: \quad \sigma=0,036$

$$
\tau=2,76 \cdot 10^{-6}
$$

strato $F_{1}: \quad \sigma=0,036 \div 0,36$

$$
\tau=5,2 \cdot 10^{-5} \div 5,2 \cdot 10^{4}
$$

strato $F_{2}$ :

$\sigma=0,073 \div 0,73$

$\tau=3,22 \cdot 10^{-3} \div 3,22 \cdot 10^{-2}$

Si noti che i valori numerici assunti per il $\operatorname{grad} T$ sono verosimilmente valori massimi cosicché è lecito ritenere $i$ valori corrispondenti di $p$ come limiti superiori.

Data la definizione di $\tau$, risulta che, anche nel caso della [18], esso ha, a parità di $\sigma$, gli stessi valori numerici nei due casi della sem. plice ricombinazione o del semplice attaccamento. 
Possiamo pertanto affermare che la correzione dovuta alla diffusione è, anche nel caso di atmosfera non isoterma, senz'altro trascurabile per gli strati $E$ ed $F_{1}$; non cosi può essere invece, almeno a priori, per lo strato $\boldsymbol{F}_{\mathbf{2}}$.

Paragrafo 2. - Procederemo ora alla soluzione delle equazioni [16] e [18]. Allo scopo di confrontare i nostri risultati con quelli ottenuti da Ferraro (per il solo caso della ricombinazione), risolveremo le equazioni stesse per un giorno equinoziale all'equatore, assumendo che sia $\sigma=1, r=-2,1+p z^{*}=2, \alpha_{T}=0,3$.

In considerazione della piccolezza del parametro $\tau$ potremo usare nella integrazione delle [16] e [18] il classico metodo delle perturbazioni sviluppando la $\nu$ in serie di funzioni rispetto al parametro $\tau$.

Ponendo

$$
v=\sum_{0}^{\infty} \tau^{k} v_{k}
$$

e sostituendo nelle [16] e [18] otteniamo, rispettivamente, le seguenti equazioni cui obbediscono le singole $\nu_{k}$

$$
\begin{aligned}
& \sigma \frac{\partial \nu_{o}}{\partial \Phi}=F-\nu_{0}{ }^{2} \\
& \sigma \frac{\partial v_{k}}{\partial \Phi}+2 v_{0} v_{k}=-\sum_{1}^{k-1} v_{k-b} v_{h}+\left(1+p z^{*}\right)^{-r-1 / 2} v^{-r+3 / 2}\left[\frac{\partial^{2} v_{k-1}}{\partial v^{2}}+\right. \\
& \left.+\frac{G_{1}}{v} \frac{\partial^{2} v_{k-1}}{\partial v}+\frac{G_{0}}{v^{2}} v_{k-1}\right] \quad \text { per } k>1 \\
& \sigma \frac{\partial \nu_{o}}{\partial \Phi}=F-\nu_{o} \\
& \sigma \frac{\partial v_{k}}{\partial \Phi}+v_{k}=\left(1+p z^{*}\right)^{-r-1 / 2} v^{-r+3 / 2}\left\lceil\frac{\partial^{2} v_{k}-1}{\partial v^{2}}+\frac{G_{1}}{v} \frac{\partial v_{k}-1}{\partial v}+\frac{C_{2}}{v^{2}} v_{k-1}\right] \\
& \text { per } k>1
\end{aligned}
$$

Come si vede dalle [20], nel caso della ricombinazione, a parte l'equazione che dà la funzione $v_{0}$ che non è lineare, tutte le equazioni nelle $\nu_{k}$ per $k \geqslant 1$ sono lineari; nel caso dello attaccamento invece tutte le equazioni [21] sono lineari. Dal punto di vista concettuale pertanto tutte le equazioni [20] e [21] tranne una sono risolubili per quadrature; il calcolo effettivo è però assai laborioso. La piccolezza 
del parametro $\tau$ consente però di arrestare lo sviluppo [19] al termine lineare in $\tau$ stesso.

I risultati del calcolo effettuato in tale ipotesi semplificativa sono riassunti nelle figg. 1 e 2 che danno gli andamenti della funzione $v_{k}$ per vari valori di $v$ : tenuto conto dell'ordine di grandezza del para-

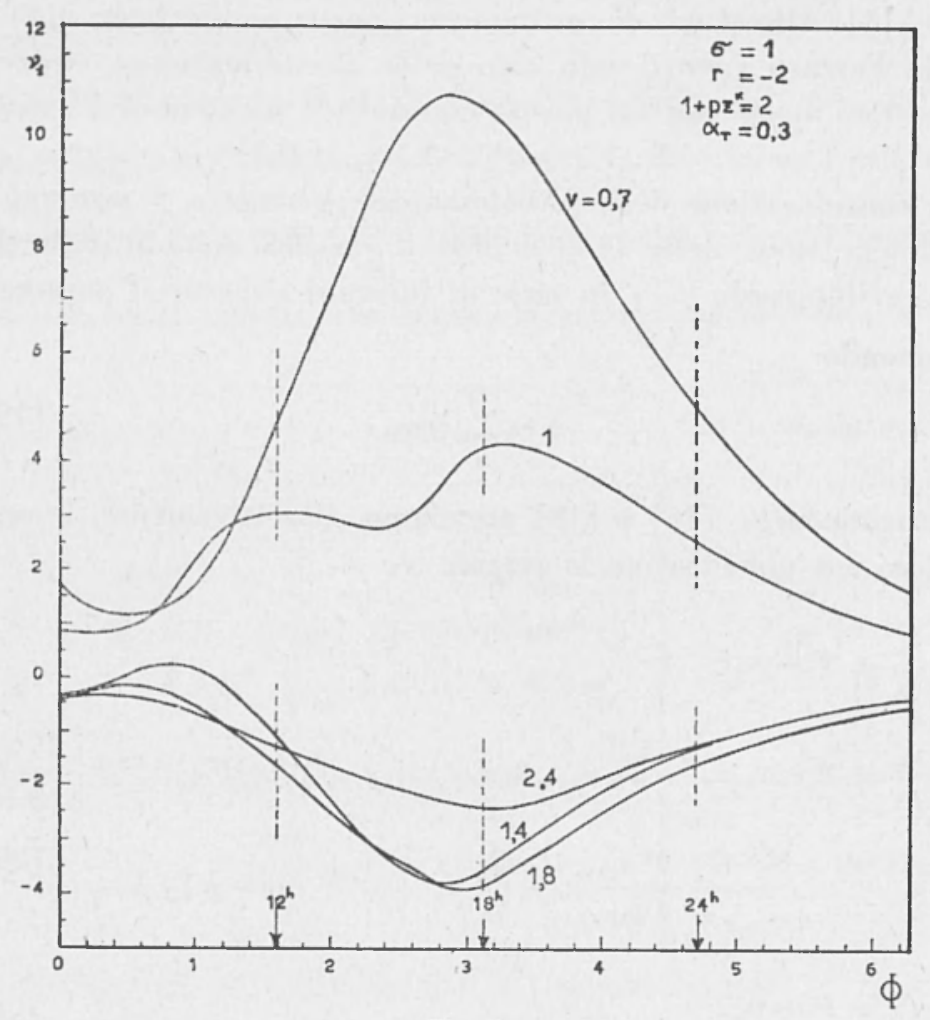

Fig. 1

metro $\tau$ risulta confermato che effettivamente le correzioni possono essere apprezzabili solo per lo strato $F_{\%}$, sia per il caso della ricombinazione che per quello dell'attaccamento.

Si vede come nel caso dell'attaccamento (fig. 1) la $v_{1}$ assume valori quasi soltanto negativi già per $v=1,4$ mentre, nel caso della ricombinazione (fig. 2), essa si mantiene positiva per tutti i valori di $v$ che abbiamo considerato; questo fatto, evidentemente dovuto alla forma stessa delle equazioni $[20]$, rappresenta la maggiore differenza qualitativa rispetto ai risultati ottenuti da Ferraro: l'andamento del calcolo nu- 
merico di $\nu_{1}$ mostra però, chiaramente, che per valori di $v$ alquanto maggiori di 2,4 la $\gamma_{1}$ viene ad assumere, anche nel nostro caso, valori negativi; comunque tale questione non è, al momento, di grande inte-

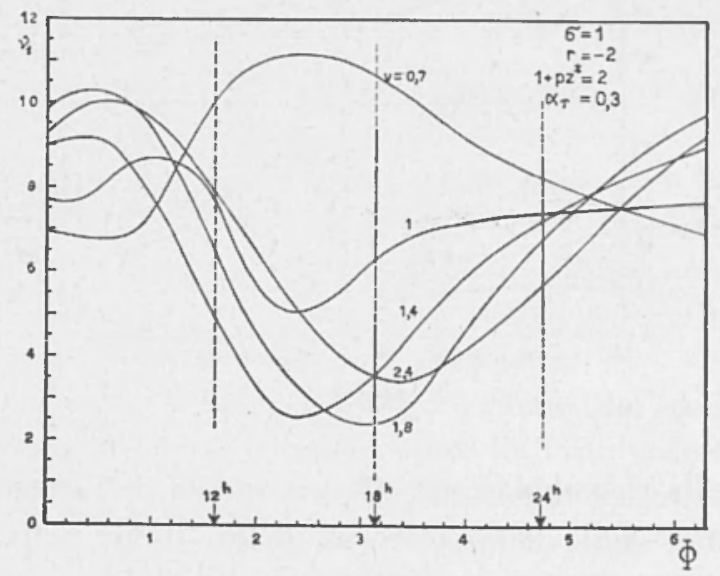

Fig. 2

resse dato che sperimentalmente, facendo astrazione dalle sporadiche osservazioni ottenute mediante razzi, è possibile "vedere" solo la parte di strato situata al disotto del massimo di densità elettronica.

Più interessanti appaiono le figg. 3 e 4 che danno l'andamento

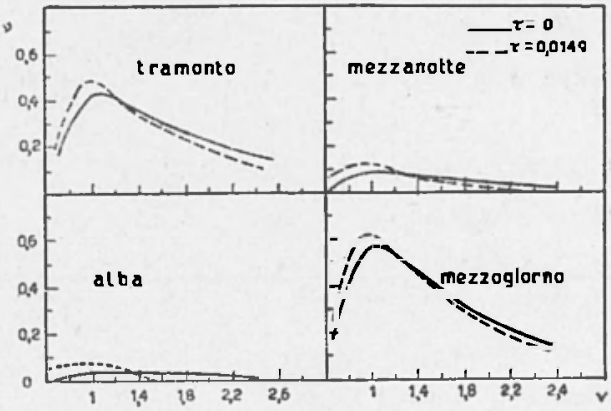

Fig. 3

di $v_{o}$ (linee a tratto continuo) e di $v_{0}+\tau v_{1}$ per $\tau=0,0149$ e $\tau=0,00664$, nel caso dell'attaccamento (fig. 3) e della ricombinazione (fig. 4), in quattro ore tipiche; $i$ valori di $\tau$ corrispondono, nel nostro caso $\sigma=1$ a $\operatorname{grad} T=7,27^{\circ} \mathrm{K} / \mathrm{km}$ e $4,85^{\circ} \mathrm{K} / \mathrm{km}$ rispettivamente. Si constata dall'esame di tali figure che l'aumento $\tau \nu_{1}$ di densità elettronica è, sia 


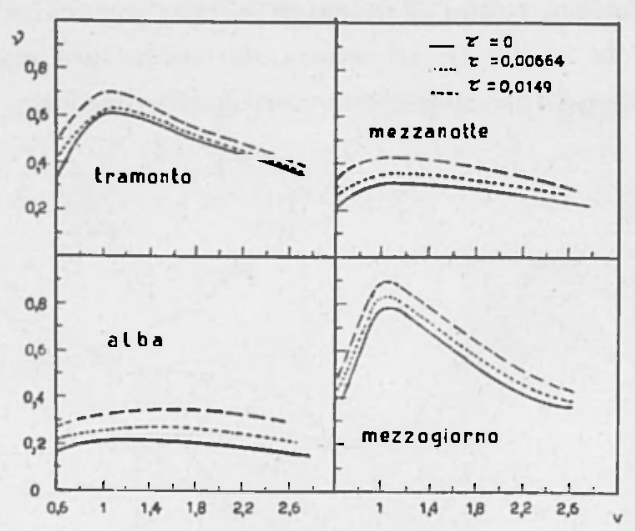

Fig. 4

per il caso della ricombinazione che per quello dell'attaccamento, più o meno notevole nella parte inferiore dello strato; nella zona al disopra del massimo, invece, la densità effettiva $v_{0}+\tau v_{1}$ scende rapida-

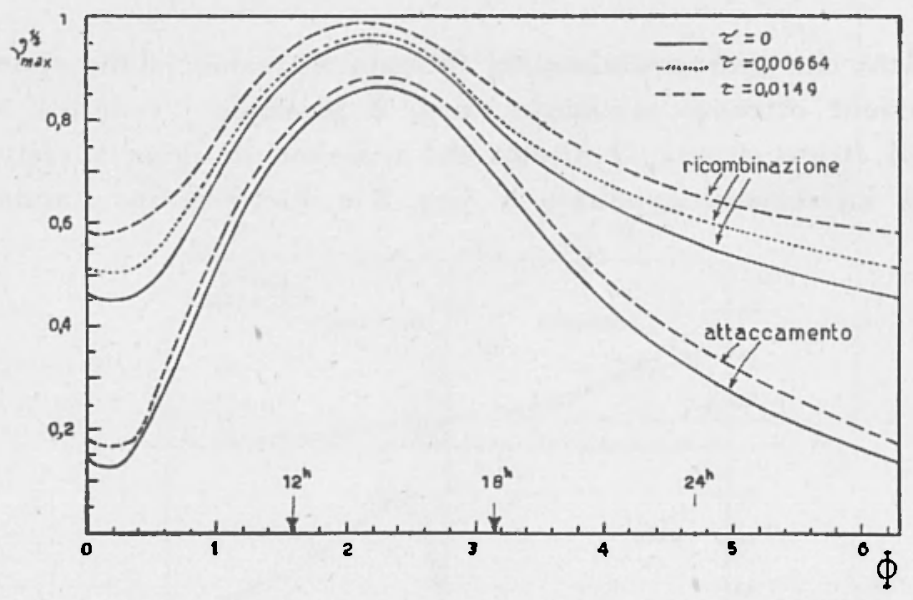

Fig. 5

mente a valori inferiori a quelli di $v_{0}$ nel caso dell'attaccamento mentre nel caso della ricombinazione essa si mantiene superiore a quella $v_{0}$ con tendenza a scendere a valori inferiori solo a quote molto alte. In quanto alla quota di massima densità essa non subisce variazioni rilevanti: è evidente una diminuzione sistematica nel caso dell'attaccamento, maggiore intorno all'alba e di entità di circa $0,5 v$; nel caso della ricombinazione invece non si hanno variazioni sensibili 
tranne che in prossimità dell'alba allorché si ha un innalzamento dell'entità di circa $0,7 v$. Tale tipo di comportamento della densità elettronica si riproduce con modalità e con entità di uguale ordine di grandezza anche nel caso $\sigma=\frac{1}{5}$ che pure abbiamo analizzato.

Nella fig. 5 , infine, sono riportati per i vari casi considerati nelle fig. 3 e 4 gli andamenti di $\left(v_{\max }\right)^{1 / i}$ che, come è noto, sono proporzionali alle frequenze critiche; si vede che in generale si ha un aumento di $\left(v_{\max }\right)^{1 / 2}$ più sensibile nelle ore notturne e in prossimità dell'alba, e di valore assoluto maggiore nel caso della ricombinazione che in quello dell'attaccamento ma circa dello stesso ordine se riferito ai valori di $\left(v_{o, \max }\right)^{1,2}$ che si hanno in assenza di diffusione.

Fin qui non abbiamo considerato l'effetto del campo magnetico terrestre: ci si convince facilmente, come ha fatto vedere Ferraro, che ove la densità di particelle sia sufficientemente piccola perché si possano ritenere gli ioni liberi di descrivere una spirale intorno alle linee di forza del campo magnetico terrestre, allora la diffusione prende luogo non lungo la verticale, come fin qui noi abbjiamo ammesso, ma lungo la direzione delle linee di forza del campo: le equazioni [16] e [18] restano valide ma il coefficiente di diffusione $D_{12}$ (e quindi il parametro $\tau$ ) va moltiplicato per $\operatorname{sen}^{2} I$ essendo $I$ l'inclinazione magnetica del luogo. Ove invece la frequenza di collisione tra molecole e ioni sia abbastanza grande rispetto alla frequenza di rotazione intorno alla direzione delle linee di forza, allora è possibile una diffusione trasversale alle linee stesse e l'effetto del campo magnetico terrestre risulta nel complesso trascurabile.

Tenuto conto di quanto sopra, essendo l'eventuale influenza del campo magnetico terrestre tale da ostacolare più o meno sensibilmente la diffusione verticale, possiamo concludere che i risultati ottenuti rappresentano un limite superiore per l'effetto della diffusione; in altri termini è probabile che l'effetto della diffusione sia, anche nel caso di atmosfera non isoterma, un semplice effetto perturbativo.

Roma - Istituto Nazionale di Geofisica - Settembre 1955. 


\section{RIASSUNTO}

Nella presente nota si studia la diffusione degli elettroni (e degli ioni) in atmosfera non isoterma. Considerando la atmosfera come costituita da un miscuglio binario (un gas ioni-elettroni e un gas di molecole neutre) si stabilisce l'espressione generale della equazione di scomparsa degli elettroni nella ionosfera includente gli effetti della diffusione. Si risolve l'equazione stessa nel caso particolare di temperatura linearmente $e$ indefinitamente crescente con la quota e si mostra che, similmente a quanto ottenuto da vari autori che hanno studiato il caso di temperatura costante a ogni quota, gli effetti della diffusione cominciano ad essere apprezzabili solo nello strato $\mathrm{F}_{2}$. Tuttavia, anche in queste condizioni e considerando che il calcolo numerico è stato condotto assumendo valori numerici che sono verosimilmente limiti superiori per il gradiente di temperatura e trascurando l'effetto del campo magnetico terrestre (che è tale da limitare più o meno considerevolmente la diffusione verticale), si conclude che la diffusione va probabilmente considerata come un effetto perturbativo che influisce solo debolmente sulla distribuzione di densità elettronica.

\section{SUMMARY}

In this paper we consider the problem of the ion-electron diffusion in a not-isotherm atmosphere; this problem has been studied by some Authors always in the case of an isotherm atmospere.

The interest for the question is due to the fact that, if, as one generally assumes, the temperature-gradient in the $\mathrm{F}$ layer is not zero, the effect of the diffusion may be more or less affected by the thermal diffusion.

We have considered the atmosphere as a binary mixture (a ionelectron gas and a molecular gas) and have obtained the general term expressing the diffusion effects for a not-isotherm atmosphere: the solution of the corresponding equation regulating the electron density is very aifficult; however, if one considers the case of temperature linearly increasing with the height, the general equation assumes the more simple expressions [16] and [18], respectively for the recombination case and for the attachment case; at last, the parameter $\tau$ 
defined by [17] is very small for the conditions of the $\mathbf{E}$ and the $\mathbf{F}_{1}$ layers and begins to be perceptible only for the conditions of the $\mathbf{F}_{2}$ layer so that we can use the perturbation theory.

The numerical results are obtained for an equinotial day at the equator: one sees that the effect of the diffusion is an increase of the maximum electron density $v_{\max }$ with respect to the case of no diffusion, more marked during the night and near sunrise; the height at which $\nu_{\max }$ occurs does not vary greatly du:ing the day with respect to the case of no diffusion: in the attachment case there is a light systematic diminution more marked near sunrise; in the recombination case there are not perceptible variations except near sunrise when the height of $v_{\max }$ rises somewhat. With regard to the effects of the terrestrial magnetic field on the diffusion it is easily seen that they may be a limitation of the transverse and hence of the vertical diffusion; however, this limitation is not effective if the collision frequency of the ions is rather large compared with the spin of a ion spiralling round a magnetic line of force. If moreover one considers that the values we have assumed for $\tau$ are likely higher limits, we conclude that the effects of the diffusion are at once negligible in the $\mathrm{E}$ and $\mathrm{F}$ layers; in the $\mathrm{F}_{2}$ layer, instead, they are likely small, also in the case of rather large temperature-gradient, so that one can consider them only as perturbative effects.

\section{BIBLIOGRAFIA}

(1) Hulburt E. O., Phys. Rev., $31-1018$ (1928).

(2) Bnadbury N.E., Terr. Magn., 43-55 (1938).

(3) Ferraro V. A., Terr. Magn., 50-215 (1945) e 51-427 (1946).

(4) Yonezawa T., J. Radio Res. Labor., 2-125 (1955).

(i) Mariani F., Ann. Geofis., 9-245 (1956).

(6) Hirschfelder J. O., Curtiss Ch. F., Bird B.R., Molecular theory of gases and liquids (1954). 